\title{
Auf der Suche nach der neuen Mittelklasse - Replik auf Nils Kumkar und Uwe Schimank
}

\section{Gesellschaftstheorie und Sozialstrukturanalyse}

Die Sozialstrukturanalyse gehört mitten in die Gesellschaftstheorie. Ohne sie kann man keine informative (Gesellschafts-)Theorie der Moderne und auch keine Theorie der spätmodernen Gesellschaft betreiben. Wenn ich in meinem Buch Die Gesellschaft der Singularitäten ${ }^{1}$ und der darauffolgenden Essaysammlung Das Ende der Illusionen ${ }^{2}$ einen Beitrag zur Gesellschaftstheorie der Moderne, genauer: zur Analyse des Strukturwandels von der industriellen Moderne zur Spätmoderne liefern möchte, dann ist für mich diese Einbettung der Sozialstrukturanalyse in die Gesellschaftstheorie ein zentraler Aspekt. Natürlich: Gesellschaftstheorie ist deutlich mehr als Sozialstrukturanalyse. Es geht ihr auch um eine Analyse der Strukturdynamiken der Ökonomie, des Staates und der Technologien sowie des kulturellen Wandels. Diese spätmodernen Strukturdynamiken - der Aufstieg des kognitiv-kulturellen Kapitalismus und des polarisierten Postindustrialismus, die Auswirkungen der Digitalisierung und der Wandel der Politik in Richtung eines apertistischen Liberalismus - und der kulturelle Wandel in Richtung einer Kultur der Singularitäten stehen so im Zentrum meiner beiden Bücher. Aber: Ohne eine Verknüpfung dieser Transformationsprozesse mit einer Analyse der sozialen Gruppen, die diesen Wandel forcieren (oder ihm entgegenstehen), die kulturelle Lebensformen ausbilden und sich diesbezüglich voneinander unterscheiden, die langfristig materiell und symbolisch auf- oder absteigen und schließlich Einfluss gewinnen oder verlieren, bliebe die Gesellschaftstheorie ein Torso. Das heißt aber: Wenn ich die »neue " und die »alte " Mittelklasse behandelt habe - jene Frage, die Nils Kumkar und Uwe Schimank kritisch thematisieren -, dann ist dies nicht isoliert als ein Anliegen der Sozialstrukturanalyse zu verstehen, sondern innerhalb dieses weiteren Rahmens einer Theorie spätmoderner Gesellschaft zu begreifen.

Es war die große Errungenschaft der Klassiker Marx und Weber, in ihren gesellschaftstheoretischen Ansätzen eine solche analytische Vermittlung von systemischen, institutionellen und kulturellen Prozessen einerseits, sozial-kulturellen Trägergruppen andererseits zu leisten. In den letzten Jahrzehnten ist diese Kopplung jedoch offensichtlich nicht mehr selbstverständlich gewesen. Die in Deutschland lange Zeit prägenden gesellschaftstheoretischen Großentwürfe von Jürgen Habermas und Niklas Luhmann meinten letztlich, ohne einen Blick auf Trägergruppen und Sozialstruktur auszukommen. Auch viele inhaltlich enger orientierte Ansätze

1 Reckwitz 2017.

2 Reckwitz 2019.

Leviathan, 49. Jg., 1/2021, S 33-7trow, DOI: 10.5771/0340-0425-2021-1-33 
zu einer Theorie spätmoderner Gesellschaft - etwa jene von Anthony Giddens, Zygmunt Bauman oder Manuel Castells - blieben in dieser Hinsicht recht wortkarg. Die Gesellschaftstheorie hat aus meiner Perspektive jedoch eine Integrationsaufgabe. Sie muss die Analyse von sozialen Großgruppen und ihrer Lebensformen als unverzichtbaren Bestandteil in die Analytik der Moderne integrieren. ${ }^{3}$ Aber auch darüber hinaus gilt: Wenn die Gesellschaftstheorie zu beschreiben und zu erklären versucht, wie sich die westlichen Gesellschaften insgesamt transformieren, muss sie, so gut es geht, die relevanten spezialisierten Informationen aus der Wirtschaftssoziologie, der Politischen Soziologie, der Techniksoziologie, der Kultursoziologie, der Bildungssoziologie etc. synthetisieren und in ein begrifflich zugespitztes Modell eines nachvollziehbaren Zusammenhangs bringen. Genau dies war in den beiden Büchern mein Ziel.

Eine solche zugespitzte Synthese hinsichtlich der Gesellschaft als Ganzer ist zweifellos ein riskantes Unterfangen. Insofern ist es grundsätzlich nicht überraschend, dass Nils Kumkar und Uwe Schimank vor dem Hintergrund ihrer eigenen qualitativ-empirischen Untersuchung zu beruflichen Orientierungen in der deutschen Mittelschicht und auch darüber hinaus zweifelnde Rückfragen anmelden, und zwar gegenüber einem besonderen Aspekt meiner Darstellung, der Frage nach der Relevanz einer neuen Mittelklasse und ihrem Verhältnis zur alten Mittelklasse.

Man sollte dabei nicht vergessen, dass die Gesellschaftstheorie hier vor einer ganz grundsätzlichen Herausforderung steht: ${ }^{4}$ Wenn sie eine begrifflich zuspitzende Synthese anvisiert, muss sie sich um die zwangsläufig schwierige Balance zwischen Abstraktion und Konkretion, zwischen allgemeinen Aussagen und empirischer Vielgestaltigkeit bemühen. Einerseits kann es nicht darum gehen, lediglich additiv die Informationen aus einer überbordenden Vielzahl von empirischen Untersuchungen in ein lockeres Gesamtbild zu verweben. Abgesehen davon, dass immer eine Selektion nötig sein wird, kann man von der Gesellschaftstheorie mehr als eine solche facettenreiche, aber letztlich unverbundene Gesellschaftsbeschreibung erwarten. Sie muss theoretisch-begrifflich vielmehr zuspitzen, eine begründete Auswahl zwischen Zentralem und weniger Bedeutsamen treffen, sie muss versuchen, nachvollziehbare Zusammenhänge zwischen verschiedenen Bereichen aufzuzeigen und Dynamiken nicht nur zu beschreiben, sondern auch zu erklären.

Gesellschaftstheorie bedeutet damit immer theoretische Reduktion von empirischer Komplexität, um umgekehrt die Komplexität einer Theorie aufbauen zu können. Sie hat etwas von einem Puzzlespiel, bei dem man am Anfang nicht weiß, wie die Teile zueinander passen, und etwas von einem begrifflich-theoretischen Experiment, bei dem man ausprobiert, wie man die empirischen Sachverhalte mit welcher Theorie am besten aufschließen kann. Es ergibt sich damit jedoch zweifel-

3 Einige Ansätze haben dies in den letzten Jahrzehnten jedoch getan, hier sind vor allem jene von Pierre Bourdieu und Gerhard Schulze zu nennen.

4 Eine genauere Darstellung dieser Aufgaben der Gesellschaftstheorie findet sich in Reckwitz, Rosa 2021. 
los ein gegenteiliges Risiko, nämlich dasjenige, dass die Theorie der Moderne »über den Wolken fliegt «, dass sie so abstrakt, selbstbezüglich und überpointiert wird, dass sie sich um die Empirie kaum mehr kümmert und sie diese als bloßen Stichwortgeber behandelt. Insofern kann man zweifellos von der Gesellschaftstheorie verlangen, dass sie sich, so gut es geht, auf die empirischen Studien zu den verschiedensten Teilbereichen der Gesellschaft einlässt. Dabei ist sie allerdings darauf angewiesen, dass bereits aussagekräftige empirische Studien vorliegen: Die Gesellschaftstheorie ist hier immer in der Rolle der »Zweitauswerterin «. Sie kann allerdings auch neuen empirischen Studien einen Impuls geben.

Also: Von der Gesellschaftstheorie muss man in jedem Fall erwarten, dass sie anhand bestehender empirischer Studien zu Einzelphänomenen Plausibilität gewinnt, zugleich ist sie zwangsläufig diesen gegenüber selektiv und spitzt sie »übertreibend « $\mathrm{zu}$ - in einer Weise, die notwendigerweise umstritten sein muss, da sie das, was vielgestaltig ist, in ein konsistentes Modell und in das Narrativ einer Prozesslogik einfügt.

\section{Soziale Großgruppen: Abstraktionsebenen und Unschärfen}

In Die Gesellschaft der Singularitäten habe ich nur ansatzweise ein Modell der spätmodernen Sozialstruktur skizziert. Der sozialstrukturell wichtigste Aspekt in diesem Buch ist in seinem fünften Kapitel eine genauere Charakterisierung der Lebensform der »neuen Mittelklasse«. Daneben interessierten mich hier auch die Auf- beziehungsweise Abwertungsprozesse, die zwischen dieser neuen Mittelklasse und einer neuen Unterklasse ablaufen. Dies alles ist eingebettet in die grundsätzliche Analyse von Prozessen der Singularisierung und Valorisierung sowie der Entsingularisierung und Entvalorisierung in der Spätmoderne. Im zweiten Kapitel meines Aufsatzbands Das Ende der Illusionen führe ich verschiedene Aspekte zur Sozialstruktur im grundsätzlicher angelegten Modell einer Drei-pluseins-Klassenstruktur weiter. Auch dies ist zweifellos eine Skizze, die man auf konzeptueller Ebene weiterentwickeln und präzisieren muss. Und vor allem: Es sind weitere empirische Studien nötig, um die offenen Fragen, die sich daraus ergeben, zu behandeln. Hinsichtlich dieses weiteren Empiriebedarfs bin ich mir mit Kumkar und Schimank ganz einig. Und ich bin dankbar, dass sie sich die Mühe einer kritischen Lektüre meiner Texte gemacht haben. Ich will aber nun die Gelegenheit nutzen, um zumindest einige Missverständnisse auszuräumen, andere Dinge zu präzisieren und dabei auch genauer auf die von mir verwendete SINUS-Studie einzugehen, als ich es bisher getan habe.

Natürlich muss man sich die generelle Aussagekraft eines soziologischen Modells vergegenwärtigen, welches soziale Klassen und ihre Lebensformen unterscheidet. Ist es nicht zwangsläufig zu "grobschlächtig " und bedarf erheblicher Differenzierung? Gibt es nicht Zwischenzonen und abweichende Fälle, die sich nicht ohne Weiteres einfügen? Kumkar und Schimank geben dies zu bedenken. Mehr Differenzierung einzufordern ist auf den ersten Blick ein Wunsch, dem man sich kaum verweigern kann. Allerdings: Jede soziologische Unterscheidung von 
sozialen Großgruppen bewegt sich im Spannungsfeld zwischen Abstraktion und Konkretion. Das eine Extrem wäre, jedes einzelne Individuum in der Unvergleichlichkeit seines Lebens zu betrachten (was etwa in der Psychotherapie versucht wird), das andere Extrem, hochabstrakt Aussagen über »die « Lebensform der westlichen Gesellschaften zu Beginn des 21. Jahrhundert zu formulieren (was etwa im Rahmen einer historischen Typologie Sinn macht). Zwischen diesen beiden Extremen bewegt sich die Unterscheidung sozialer Großgruppen. Auch diese kann nun entweder um eine relativ große Differenzierung bemüht sein, das heißt zum Beispiel eine größere Anzahl von - zehn? zwanzig? - Milieus unterscheiden, die immanent in ihrer Lebensweise recht homogen erscheinen. Oder aber man bemüht sich um eine größere Abstraktion, sodass mehrere kleinere Milieus auf abstrakterer Ebene als verschiedene Ausprägungen eines übergreifenden Wertesystems oder eines übergreifenden sozialen Status interpretiert werden können und man in der Gesellschaft einige wenige zentrale Kräfte des sozialen Wandels unterscheidet. Im Extrem hatte dies Marx mit seiner Gegenüberstellung von Bourgeoisie und Proletariat getan. Beide Strategien haben offenkundige Vor- und Nachteile. Was sich besser eignet, hängt letztlich von der Fragestellung ab.

Mit meinem Vorschlag einer Differenzierung von Oberklasse, neuer Mittelklasse und alter Mittelklasse sowie Unterklasse wähle ich im gesellschaftstheoretischen Rahmen eine vergleichsweise hohe Abstraktionsebene, da sich auf diese Weise großflächige Wandlungsprozesse und Konfliktkonstellationen am besten begreifen lassen. Die Pointe dieser zunächst recht klassischen Unterscheidung zwischen »Oben «, »Mitte « und »Unten « besteht tatsächlich in der Gegenüberstellung der beiden Mittelklassen. Dieses Modell ist aber keinesfalls als Ablehnung von detaillierteren und differenzierten Milieustudien zu lesen, im Gegenteil. Am klügsten ist aus meiner Sicht folgende Annahme: Für eine Analyse sozialer Großgruppen der Gegenwartsgesellschaft sollte man drei Abstraktionsebenen voneinander unterscheiden, die je nach Fragestellung flexibel miteinander zu kombinieren sind. Auf einer ersten Ebene lassen sich vergleichsweise kleine und daher verhältnismäßig viele, in sich recht homogene Milieus voneinander unterscheiden - das sind etwa die SINUS-Milieus. Auf einer zweiten, abstrakteren Ebene lassen sich größere sozialkulturelle Klassen anhand allgemeinerer Merkmale unterscheiden, so wie es bei mir im Zentrum steht (sodass umgekehrt eine einzelne Klasse mehrere Milieus auf der 1. Ebene »umfasst «). Auf einer dritten, noch abstrakteren Ebene werden Gemeinsamkeiten beziehungsweise Homologien zwischen verschiedenen Klassen sichtbar, zum Beispiel zwischen neuer Mittelklasse und alter Mittelklasse oder zwischen neuer Mittelklasse und neuer Oberklasse etc. (oder gar zwischen allen Klassen).

Ich habe mich in meiner eigenen Analyse primär auf der zweiten Ebene bewegt. Grundsätzlich wird man jedoch alle drei Ebenen betrachten müssen. Ich denke, dass manche Einwände gegen das Modell der neuen Mittelklasse, die auch Kumkar und Schimank erheben, sich dadurch begegnen lassen, dass man tatsächlich alle drei Ebenen in den Blick nimmt und sie nicht gegeneinander ausspielt. Denn dann zeigt sich nämlich einerseits, dass die neue Mittelklasse (so wie übrigens auch die alte) nach innen zweifellos kulturell und sozial differenziert und hetero- 
gen ist und sich in mehreren unterschiedlichen kulturellen Milieus konkretisiert. Sie ist auch, was Einkommen und Vermögen angeht, differenziert (was nicht im Zentrum meiner Betrachtungen steht) und sicher auch unter anderen relevanten Gesichtspunkten, was etwa Generationen oder Alterskohorten oder selbst die Bildung angeht. Andererseits zeigt sich, dass - genau umgekehrt - auf höherer Abstraktionsebene auch Homologien nicht nur zwischen manchen Milieus, sondern ebenso zwischen bestimmten Klassen existieren. Namentlich zwischen der neuen und der alten Mittelklasse lassen sich so abstrakte Gemeinsamkeiten feststellen (was auch nicht derart überraschend sein dürfte: Sie sind ja beide »Mittelklassen $\ll)$.

Auf welcher Ebene man sich auch immer bewegt, es ergibt sich allerdings ein grundsätzliches Problem der soziologischen Modellbildung sozialer Großgruppen: Gleich, wo man die Grenzen zieht, es passen nicht alle Individuen problemlos in eine Gruppe hinein, es gibt immer mehr oder minder viele Individuen, die kulturelle Merkmale mehrerer Gruppen aufweisen oder sich durch Statusinkonsistenz auszeichnen oder noch weitere wichtige Merkmale aufweisen. Gerhard Schulze hat dies in seiner Sozialstrukturanalyse sehr gut problematisiert. ${ }^{5}$ Er spricht von »Grenzfällen und Inkonsistenzen «, von sozialer »Unschärfe « und von den "Grenzzonen ", die sich zwischen den Milieus (oder in unserem Kontext: den Klassen) befinden, von »Kernbereichen und Bereichen relativer Unbestimmtheit «. In der Modellbildung der Großgruppen - ob nun bei SINUS, bei Schulze, bei Bourdieu oder bei mir - überspitzt man die Darstellung zwangsläufig und suggeriert, auch in der grafischen Darstellung, dass es klare Grenzen zwischen den Gruppen gäbe. Das ist aber zumal in den spätmodernen, im Ulrich Beck'schen Sinne individualisierten Gesellschaften nicht der Fall: Der Realität näher kommt das Bild der »Kernbereiche" von Milieus/Klassen und »Grenzzonen «. Aus der gesellschaftstheoretischen Perspektive wird man hier immer stärker zuspitzen müssen, aber man sollte sich eine Sensibilität für die Grenzfälle und Statusinkonsistenzen bewahren.

\section{Die neue Mittelklasse als Phänomen und soziologisches Thema}

Die Mehrebenenhaftigkeit sozialer Großgruppen und die Konstellation von Kernbereichen und Grenzzonen sind im Hinterkopf zu behalten, wenn wir uns nun der neuen Mittelklasse nähern. Ich möchte es noch einmal betonen: Die Herausbildung dieser neuen Mittelklasse ist aus meiner Perspektive für eine Theorie der spätmodernen Gesellschaft tatsächlich ein zentraler Aspekt. ${ }^{6}$ Die neue Mittelklasse entsteht am Kreuzungspunkt dreier seit dem letzten Drittel des 20. Jahrhunderts die gesamte Gesellschaft prägenden Transformationsprozesse: der Postindustrialisierung der Ökonomie mit der Expansion der Wissensökonomie, der Bildungsexpansion sowie des kulturellen Wertewandels, das heißt eines kulturel-

5 Schulze 1992, S. 382-386.

6 Vgl. Reckwitz 2017; Reckwitz 2019.

Leviathan, 49. Jg., 1/2021 
len Liberalisierungsprozesses, in dessen Verlauf Selbstverwirklichungswerte gegenüber Pflicht- und Akzeptanzwerten an Relevanz gewinnen.

Die Lebensform der neuen Mittelklasse stellt sich auch als zentrale Trägergruppe von Prozessen der Kulturalisierung und Singularisierung dar, wie sie für die Spätmoderne charakteristisch sind. In besonderem Maße trägt die neue Mittelklasse - ob auf der Produzenten- oder Konsumentenseite - den Aufstieg des kognitiv-kulturellen Kapitalismus und die Aufmerksamkeitsökonomie der digitalen Technologien sowie den politischen Aufstieg eines neuen Liberalismus von den $1980 \mathrm{er}$ bis zu den 2000er Jahren. Die neue Mittelklasse ist somit einerseits das Produkt bestimmter Wandlungsprozesse, und zugleich treibt sie selbst - ohne dass sie sich dessen notwendig bewusst wäre oder ein Kollektivbewusstsein ausbilden würde - diese Prozesse an.

Sie stellt sich darüber hinaus als eine Seite jenes spätmodernen sozialstrukturellen Paternosters dar, in dem den "Aufsteigern " "Absteiger « gegenüberstehen. Potenziell kann sich die soziale und symbolische Differenz zwischen der neuen Mittelklasse auf der einen Seite, der alten Mittelklasse und der neuen Unterklasse auf der anderen in kulturelle und politische Konflikte umsetzen, von denen seit 2010 einige virulent geworden sind, so der Aufstieg des Rechtspopulismus. Kurzum: Ohne die neue Mittelklasse als soziale Trägergruppe lassen sich zentrale soziale Wandlungsprozesse von der industriellen Moderne zur Spätmoderne wie auch Konfliktkonstellationen der Spätmoderne nicht verstehen. Ich würde durchaus mit einer gewissen Emphase behaupten, dass wir in der Soziologie unseren Blick auf die Gegenwartsgesellschaft deutlich schärfen, wenn wir uns entschließen, die Auswirkungen der neuen Mittelklasse in verschiedensten Spezialsoziologien - ob in der Politischen Soziologie oder in der Wirtschafts- und Arbeitssoziologie, ob in der Kultursoziologie oder der Erziehungssoziologie, ob in der Stadtsoziologie oder der Soziologie des Körpers - stärker zu berücksichtigen, als wir dies bisher getan haben.

Vier allgemeine Merkmale habe ich für die neue Mittelklasse hervorgehoben: Erstens und im Zentrum steht ein Wertesystem und eine entsprechende soziokulturelle Praxis, die im weiteren Sinne Selbstentfaltungswerte und postmaterialistische Werte - Selbstverwirklichung, "gutes Leben « und "innere Balance ", Bedeutung von Ethik (Nachhaltigkeit, Ökologie) und Ästhetik, Individualität, Authentizität, Singularisierung des Alltags, Offenheit, Entgrenzung, Kreativität, Liberalität und Diversität etc. - umfassen. Vereinfacht können wir hier vom Wertecluster eines expressiven Individualismus sprechen. Dieses verbindet sich mit einem Interesse an Statusinvestition, Statussicherung und Kapitalakkumulation, sodass beide Aspekte zusammen in das kulturelle Modell einer »erfolgreichen Selbstverwirklichung " münden (ein Statusinteresse, das sich auch in Zielen wie Gesundheitsorientierung, Mobilität, lebenslangem Lernen oder Flexibilität konkretisiert).

Zweitens verfügt die neue Mittelklasse über überdurchschnittlich viel kulturelles Kapital, die Individuen haben in der Regel höhere Bildungsabschlüsse wie einen Hochschulabschluss, was sich meist in entsprechend höher qualifizierte Berufstätigkeit umsetzt. Das dritte Merkmal betrifft nicht die Binnenstruktur der neuen 
Mittelklasse, sondern ihr Verhältnis zur Gesamtgesellschaft: Es existiert ein hoher kultureller Einfluss ihrer Werte auf Politik, Wirtschaft, Kultur, Bildung, Medien etc. Die neue Mittelklasse ist trotz ihrer Neuheit eine mächtige Klasse, die in mancher Hinsicht kulturelle Dominanz erlangt hat. Zudem kann, viertens, nicht deutlich genug betont werden, dass die Analyse der neuen Mittelklasse sich keineswegs nur auf Deutschland bezieht, sondern auf die westlichen Gesellschaften insgesamt. Es handelt sich um ein internationales Phänomen - was bedeutet, dass man auch auf entsprechende internationale Quellen zurückgreifen muss.

Dass einflussreiche Teile der Mittelklasse seit den 1970er, 1980er Jahren einen Modernisierungsprozess durchlaufen haben und sich so eine neue Mittelklasse ausbildet, ist nun keine neuartige oder ungewöhnliche Einsicht, sondern ein Thema, das die Soziologie seit längerem beschäftigt. Dass die Diagnose der neuen Mittelklasse bei manchen in der deutschen Soziologie der Gegenwart Verwunderung hervorruft, muss daher überraschen. Zur Erinnerung: Daniel Bell hatte in The Coming of the Post-Industrial Society ${ }^{7}$ bereits Anfang der 1970er Jahre auf die neue soziale Großgruppe der Wissensarbeiter (knowledge worker) mit hoher kognitiver Kompetenz hingewiesen. Pierre Bourdieu zeigte in Die feinen Unterschiede $^{8}$ innerhalb der Mittelklasse den Aufstieg einer neuer Subklasse auf, die er "neues Kleinbürgertum " nannte und in Berufen der Kreativ- und Wissensökonomie ausmachte. Beeinflusst sowohl von Bourdieu als auch den Theorien der postmodernen Kultur, hat der Kultur- und Konsumsoziologe Mike Featherstone dieses neue Kleinbürgertum als kulturelles Leitmilieu der postmodernen Gesellschaft interpretiert. ${ }^{9}$

Seit den 1970er Jahren hat im Gefolge von Ronald Ingleharts breit rezipierten Studien zum Wertewandel (The Silent Revolution ${ }^{10}$ ) die Analyse eines "postmaterialistischen " Milieus insbesondere in der Politikwissenschaft eingesetzt. Paul Leinberger und Bruce Tucker unterziehen in The New Individualists ${ }^{11}$ den Lebensstil und die Kultur des Selbst eines spätmodernen expressiven Individualismus im Akademikermilieu einer sehr detaillierten qualitativ-empirischen Untersuchung. Es gibt auch systematische und quantitativ-standardisierte Untersuchungen wie Gerhard Schulzes groß angelegtes Buch Die Erlebnisgesellschaft, ${ }^{12}$ in dem das Selbstverwirklichungsmilieu der jüngeren Gebildeten das geheime Zentrum der Analyse darstellt, und Richard Floridas The Rise of the Creative Class, in dem die »kreative Klasse" als Motor der kulturellen und wirtschaftlichen Entwicklung erscheint. ${ }^{13}$ In jüngeren Untersuchungen, so in Robert Putnams Our Kids,${ }^{14}$ wird

7 Bell 1973.

8 Bourdieu 1989.

9 Featherstone 1991.

10 Inglehart 1977.

11 Leinberger, Tucker 1991.

12 Schulze 1992.

13 Florida 2002.

14 Putnam 2015.

Leviathan, 49. Jg., 1/2021 
die Lebensform der gut ausgebildeten Mittelklasse regelmäßig den Abgehängten in der neuen Unterklasse gegenübergestellt, die somit zwei konträre Seiten der Gegenwartsgesellschaft darstellen. Mittlerweile existiert auch eine Reihe qualitativer Detailstudien zu einzelnen Aspekten dieser neuen Klasse, ob zum Konsum oder zur Erziehung, zu Sport und Ernährung oder zum Wohnen. ${ }^{15}$

\section{Die neue Mittelklasse und die SINUS-Milieus}

Die bereits existierenden Analysen zur neuen Mittelklasse überlappen sich zwar, aber beziehen sich nicht immer exakt auf die gleiche Gruppe. Auch die empirischen Grundlagen sind sehr unterschiedlich. Gerade deshalb habe ich noch einmal begrifflich neu angesetzt und zugleich ein sozialstrukturelles Gesamttableau versucht. Mit dem vorgeschlagenen Begriff der neuen Mittelklasse versuche ich eine zugespitzte Synthese im Rahmen der Gesellschaftstheorie. Was wenden Kumkar und Schimank dagegen ein? Dass es eine solche soziale Gruppe von höher Gebildeten, Selbstverwirklichungswerte und Statusziele vertretenden Individuen gibt, bestreiten auch die beiden Kommentatoren nicht. Neben der offenen Frage der internen Differenziertheit dieser Gruppe stellen sie aber vor allem ihren quantitativen Umfang infrage. Überschätze ich - und auch viele der anderen genannten Autoren - die Relevanz der neuen Mittelklasse?

Nun ist Relevanz nicht nur eine quantitative, sondern auch eine qualitative Frage: Eine Gruppe kann, was die Zahl ihrer Köpfe angeht, durchaus von begrenzter Größe, aber ihre kulturellen Muster können hoch einflussreich und gesellschaftsprägend sein. Sie vermag dann auch ihre Interessen effektiver zu vertreten als zahlenmäßig größere Gruppen. Ein klassisches Beispiel: Das doch recht kleine Bürgertum (maximal zehn Prozent der Gesellschaft) hat im 19. Jahrhundert mit seinen Ideen und Interessen eine ganze bürgerliche Gesellschaft geprägt. Manche sozialen Gruppen sind finanzkräftiger, engagierter, mächtiger als andere, wirken als Gatekeeper für Ideen und können trotz begrenzter Zahl von Köpfen einen überproportionalen Einfluss ausüben. Man sollte sich hier nicht vom statistischen »Mythos der großen Zahl« blenden lassen - das gilt auch für die neue Mittelklasse.

Aber zweifellos: Die reine Quantität einer sozialen Gruppe ist eine wichtige Größe, man wünscht sich eine repräsentative Untersuchung, die uns diese Frage beantwortet. Die Milieustudien des Heidelberger SINUS-Instituts sind in diesem Kontext in vielerlei Hinsicht sehr fruchtbar. ${ }^{16}$ Es handelt sich um eine Kartografie kultureller Lebensstile, die erstens kultursoziologisch und qualitativ recht detailliert und reichhaltig ist und insofern informative Aussagen über Werte und kultu-

15 Vgl. etwa Currid-Halkett 2017; Lareau 2003; Harrison 2005; Arsel, Bean 2013; Wheaton 2013.

16 Ich beziehe mich im Folgenden auf: »Die SINUS-Milieus in Deutschland. Basis-Infopaket. Beschreibungen, Daten, Illustrationen ", Heidelberg, November 2015. Die im Folgenden angegebenen Seitenzahlen stammen aus diesem Bericht. Die Prozentzahlen habe ich mit Blick auf die Studie von 2020 aktualisiert. 
relle Praktiken von Großgruppen in der Bevölkerung macht, die zugleich zweitens repräsentativ ist und quantitativ den Umfang der einzelnen Milieus bestimmt, die drittens zumindest ansatzweise (wenn auch nicht absolut befriedigend) die Ressourcenausstattung der Milieus mitberücksichtigt, die viertens nicht auf Deutschland beschränkt ist, sondern international vergleichend agiert, und die fünftens den sozialen Wandel abbildet, da die Studie seit 1980 betrieben und immer wieder aktualisiert wird.

Insgesamt ziehe ich den Schluss: Die SINUS-Studie macht sehr plastisch den Modernisierungsprozess innerhalb der Mittelklasse der westlichen Gesellschaften deutlich. Es wird die Herauskristallisierung und Expansion mehrerer Milieus sichtbar, in denen in verschiedenen, konkreten Ausformungen das allgemeine kulturelle Muster jenes "expressiven Individualismus « gelebt wird, wie es die modernisierte Mittelklasse ausmacht. Es wird deutlich, wie es sich vom übergreifenden kulturellen Muster jener Milieus unterscheidet, die man als traditionelle oder alte Mittelklasse bezeichnen kann. Man erkennt zudem auch die »feinen Unterschiede « zwischen den einzelnen Milieus innerhalb der neuen Mittelklasse.

Da ich diese Einordnung des Klassenmodells in den Kontext der SINUS-Studie in Das Ende der Illusionen nur sehr knapp vornehmen konnte, ${ }^{17}$ will ich dies hier nachholen. Die SINUS-Milieus sind grafisch entlang zweier Koordinatenachsen angeordnet: Die y-Achse bezeichnet die soziale Lage, die $\mathrm{x}$-Achse die kulturelle Grundorientierung. Letztere ist eine Modernisierungsachse, durchaus im Sinne von Gerhard Schulzes »innengerichteter Modernisierung «: ${ }^{18}$ Traditionalistische und materialistische Werte stehen hier » moderneren « Werten gegenüber, die unter den Überschriften »Sein und Verändern " (Selbstverwirklichung etc.), »Machen und Erleben « (Multioptionalität etc.) und »Grenzen überwinden « (Exploration etc.) zusammengefasst werden. Hinsichtlich der sozialen Lage unterscheidet SINUS leider nicht genauer zwischen ökonomischem und kulturellem Kapital, sodass in unserem Sinne hier nur eine grobe Einordnung möglich ist.

Wenn wir im Rahmen dieser Milieuunterscheidung nach einer neuen Mittelklasse suchen, dann müssen wir aber prinzipiell in den Bereich rechts oben in der SINUS-Grafik schauen: in die höheren sozialen Lagen, die zugleich die neuen Werte vertreten. ${ }^{19}$ Man muss sich allerdings die Mühe machen, genauer in die einzelnen Milieus zu blicken. SINUS präsentiert hier für jedes Milieu leitende Semantiken und kulturelle Charakteristika. Nach Art eines Codings lassen sich nun diese speziellen Semantiken als Exemplifikationen allgemeinerer kultureller Muster interpretieren. Meine Leitfrage ist: Welche Milieus lassen sich als Exemplifikationen des Werteclusters des »expressiven Individualismus « interpretieren, welche nicht?

17 Reckwitz 2019, S. 122-125.

18 Schulze 1992, S. 41.

19 Siehe www.sinus-institut.de/sinus-loesungen/sinus-milieus-deutschland/ (Zugriff vom 03.01.2021). Ich habe selbst die SINUS-Grafik in das Klassenmodel transferiert; vgl. Reckwitz 2019, S. 124. 
Bei genauerer Betrachtung stellen sich vier Milieus als Konkretisierungsorte der neuen Mittelklasse heraus:

1. Das liberal-intellektuelle Milieu: SINUS spricht hier explizit von einem "postmateriell geprägten Individualismus « (40). »Selbstbestimmung und Selbstentfaltung, Authentizität « (40) sind hier zentrale Werte. Die »Pflege der Lebenskunst [...], subtile Genüsse, Ästhetik, Bildung, Kultur « (40) sind wichtig. Man hat eine »liberale Grundhaltung: Weltoffenheit, Toleranz, kosmopolitische Weltsicht [...], Diversity « (40), betont auch die »ethischen Grundlagen des Zusammenlebens « (40). Zugleich sind »Leistungsbereitschaft « und »Erfolgsstreben, aber keine klassische Karriereorientierung « (40) bedeutsam. Die Distinktion gilt »kleinbürgerlichen Werten « und »Konventionalität « (43). Das liberal-intellektuelle Milieu macht sieben Prozent der Gesamtbevölkerung aus.

2. Das sozial-ökologische Milieu: Hier handelt es sich um ein Milieu mit »starken Wertbezügen und postmateriellen Ansprüchen « (125), einem »ausgeprägten ökologischen und sozialen Gewissen [...], aber auch [dem] Wunsch nach Freiheit, Selbstverwirklichung, Genuss und Sinnlichkeit« (125). Das »Streben nach Authentizität, Ganzheitlichkeit und Balance« (125) ist wichtig. Man setzt auf »Nachhaltigkeit und Entschleunigung « (129) im eigenen Leben und in der Gesellschaft und grenzt sich vom schrankenlosen Materialismus und einer Kultur der Inauthentizität ab. Das sozial-ökologische Milieu umfasst sieben Prozent der Bevölkerung.

3. Das expeditive Milieu: Hier macht SINUS eine hohe "Lebens- und Experimentierfreude « (74) aus, man sei »immer auf der Suche nach neuen Erfahrungen « (74). "Grenzen durchbrechen, Horizonte erweitern « (74) sind leitende Motive des Lebens, »Weltoffenheit, Kreativität und Vitalität (74), auch globale Mobilität und soziale Vernetzung sind zentrale Werte (73). Dies ist das Milieu der Norm des Nonkonformismus. Zugleich wird das Leben von "erfolgreicher Selbstvermarktung « (74) geprägt, von hoher Ambitioniertheit, aber zugleich heißt es: »Karriere nicht um jeden Preis « (74). Die Distinktion gilt hier einem Durchschnittsleben bürgerlicher Konventionen (77). Das expeditive Milieu umfasst neun Prozent der Bevölkerung. Es ist ein vergleichsweise junges und wachsendes Milieu.

4. Das Milieu der Performer: Hier handelt es sich SINUS zufolge um die Lebensführung einer »Konsum- und Stilavantgarde (56) mit einem »kosmopolitische[n] Lebensstil « (61), in dem wiederum die Werte »Selbstverwirklichung und intensive[s] Leben " (57) hochgehalten werden und »Individualität und Freiheit als zentrale Werte (57) gelten. Den besonderen Akzent erhält dieses Milieu durch seinen starken "Leistungsoptimismus « (57) und seine »kompetitive Grundhaltung « (61), die ausgeprägte Orientierung an Flexibilität und am Globalismus. Im Laufe der Jahrzehnte ist dieses Milieu zugleich nachdenklicher geworden: "soziale Eingebundenheit [...], Nachhaltigkeit und Authentizität « (57) spielen eine zunehmende Rolle. Die Distinktion gilt generell einem genügsamen Leben der »Bequemlichkeit « und dem »Rückwärtsgewandte[n] « (60). Das Milieu der Performer macht acht Prozent der Gesamtbevölkerung aus. 
Man muss es sich klar verdeutlichen: Bei den SINUS-Milieus handelt es sich um eine repräsentative, die gesamte Bevölkerung umfassende Untersuchung. Die Aussagen dieses umfassenden Gesellschaftstableaus sind aus meiner Sicht eindeutig:

1. In den höheren sozialen Lagen der Mittelklasse und damit der Gesellschaft insgesamt existiert ein beträchtliches Segment, dessen Lebensführung von Versionen jener Werte eines expressiven Individualismus geprägt ist, die nach meinem Verständnis die "neue Mittelklasse " ausmachen und die zuvor von Autoren wie Inglehart, Featherstone, Leinberger/Tucker, Florida oder Schulze diagnostiziert wurden. Größtenteils wird der expressive Individualismus mit Motiven der Statusinvestition verbunden. Nach der SINUS-Erhebung handelt es sich in Deutschland insgesamt gegenwärtig um ein Segment von ca. 31 Prozent. Es gibt hinsichtlich dieser Zahlen sicher eine gewisse Unschärfe, aber man erhält doch eine Orientierung, von welchen Größen wir ungefähr reden. Das heißt aber auch: Quantitativ geht die Lebensführung der neuen Mittelklasse weit über jenes enge Segment hinaus, das in den Medien und darüber hinaus gelegentlich unter dem Etikett der »kosmopolitischen Eliten " firmiert. ${ }^{20}$

2. Ein Lackmus-Test für die Zugehörigkeit zur neuen Mittelklasse ist die mehr oder minder subtile Distinktion jedes dieser Milieus gegen typische Werte der klassischen, der traditionellen Mittelklasse. Tatsächlich ist für alle vier Milieus eine solche Abgrenzung gegen die vorgebliche »Enge" dieser traditionellen Mittelklasse, gegen ein Ordnungs- und Sicherheitsstreben, gegen eine Immobilität und Inflexibilität oder gegen einseitige Statusorientierung kennzeichnend. Wenn wir davon ausgehen, dass jede Klasse - mit Bourdieu - immer auch eine Distinktion nach außen übt, dann ergibt sich aus der SINUS-Untersuchung, dass die Abgrenzung der Milieus der neuen Mittelklasse eher in zweiter Linie der Unter- und der Oberklasse gilt, vielmehr sehr deutlich den Werten der traditionellen Mittelklasse und ihrer »unmodernen « Lebensführung.

3. Die Unterscheidung zwischen Milieus und Klasse erlaubt, die inneren kulturellen Differenzen innerhalb der neuen Mittelklasse zu erkennen. Diese Differenzen betreffen etwa das Verhältnis zum Konsum oder zur Leistung. Die stärkste Spannung existiert in dieser Hinsicht wohl zwischen den beiden Milieus der Performer - sehr leistungsbewusst und konsumfreudig - und der Sozial-Ökologischen - eher leistungsskeptisch und Anhänger des »ethischen Konsums «. Es ist zu vermuten, dass gerade diese Differenzen sich auch politisch abbilden lassen (Neoliberalismus vs. »links-grün«). Man kann nicht wirklich ein Kernmilieu der neuen Mittelklasse feststellen, in gewisser Hinsicht liefert jedes der vier Milieus in gesteigerter Form Eigenschaften, die insgesamt in moderierter Form in den Typus der neuen Mittelklasse eingehen: die hohe Bedeutung von Bildung und Kultur (Liberal-Intellektuelle), von Kreativität und Grenzüberschreitung (Expeditive), von Mobilität und Globalität (Performer) und von Ökologie (Sozial-Ökologische). Die immanenten Dif-

20 Vgl. etwa Strenger 2019.

Leviathan, 49. Jg., 1/2021 
ferenzen verhindern aber möglicherweise, dass sich ein Kollektivbewusstsein der neuen Mittelklasse ausbildet.

4. Die Milieu- und damit Klassenkonfiguration, die SINUS in Deutschland herausarbeitet, findet sich qualitativ und quantitativ in ähnlicher Weise auch für andere westliche Länder, in denen SINUS seine Studien durchführt. Mir liegen die Meta-SINUS-Milieus zu den USA, Großbritannien, Frankreich, Italien, Spanien, Schweden, den Niederlanden, Russland, Polen, China und Thailand vor. Es handelt sich also nicht um ein deutsches Phänomen, sondern um eines, das die klassischen Länder des Westens ebenso wie Osteuropa und schließlich in den Schwellenländern Asiens dort zumindest die Metropolregionen betrifft. Im zahlenmäßigen Umfang der neuen Mittelklasse, der traditionellen Mittelklasse und der Unterklasse ergeben sich dabei kleinere Abweichungen.

5. Im historischen Durchlauf wird deutlich, dass seit dem Beginn der SINUS-Studien 1980 bis zur Gegenwart der Umfang des Segments jener mit neuen Mittelklasse-Werten deutlich, nämlich von 13 auf 31 Prozent angestiegen ist. Das ist ein Faktum, das in seiner Tragweite nicht zu unterschätzen ist. ${ }^{21}$ Es handelt sich tatsächlich um eine allmähliche, eine silent revolution - und gerade weil sie so allmählich ist, wird sie leicht übersehen.

Man muss allerdings auch feststellen, welche Fragen zur neuen Mittelklasse sich mit SINUS nicht gut beantworten lassen und wo sich entsprechende Forschungsdesiderata ergeben. Als Hauptschwäche nehme ich hier die eher summarische Einordnung der »sozialen Lage " wahr. Die Milieus werden grafisch in »oben «, » mittel « und »unten « zugeordnet. Es werden zwar Durchschnittseinkommen und grobe Berufsverteilungen in den Milieus genannt. Es gibt aber keine expliziten Angaben zum Bildungshintergrund, man kann höchstens versuchen, anhand der Berufsverteilung diesen indirekt zu erschließen. Auch das Verhältnis zwischen ökonomischem und kulturellem Kapital in den Milieus ist so nicht transparent. Immerhin: Liberal-Intellektuelle und Performer werden von SINUS eindeutig als "höhere Mittelschicht" eingeordnet, die Expeditiven und Sozial-Ökologischen befinden sich an der Grenze zwischen hoher und mittlerer sozialer Lage. ${ }^{22}$ Insgesamt korrespondiert das mit meiner sehr vorsichtigen Annahme, dass ökonomisch die neue Mittelklasse insgesamt ein eher überdurchschnittliches Einkommen hat und dass zugleich in ihr eine beträchtliche Bandbreite existiert. Aber: Dies wüsste man - klassisch soziologisch - natürlich gerne sehr viel genauer (auch was die Vermögensverhältnisse betrifft).

Was das kulturelle Kapital angeht, würde ich nun eine differenziertere Annahme formulieren, als ich das bisher gemacht habe: Die Personen der neuen Mittelklasse

21 Dabei geht es nicht darum, zu behaupten, dass dieser Anteil vorher Null war. Das wissen wir nicht. Wenn man allerdings Ronald Ingleharts Untersuchungen zum Wertewandel hinzuzieht, ist anzunehmen, dass die Selbstverwirklichungswerte bereits in den 1970er Jahren an Fahrt aufgenommen haben.

22 Die Haushaltsnettoeinkommen von Liberal-Intellektuellen und Performern sind so überdurchschnittlich, von Sozial-Ökologischen und Expeditiven (von denen sich allerdings noch sehr viele in Ausbildung, das heißt in der Regel im Studium befinden, was die Angaben verzerrt) sind sie durchschnittlich. 
(die vier SINUS-Milieus) haben in der Regel ein überdurchschnittlich hohes kulturelles Kapital, überdurchschnittlich viele haben einen Hochschulabschluss. Dies geben die SINUS-Daten indirekt her, und das entspricht auch anderen empirischen Untersuchungen, etwa den Analysen von Ronald Inglehart zum Zusammenhang zwischen dem, was er früher postmaterialistische Werte nannte und heute sozialliberale Werte nennt, und dem Bildungsabschluss - auf die USA bezogen gerne als »College-Abschluss ja oder nein " operationalisiert. Also: Expressiver Individualismus und Bildung korrelieren positiv miteinander. Das heißt aber nicht, dass ausnahmslos jedes Mitglied der vier Milieus und damit der neuen Mittelklasse ein Akademiker oder eine Akademikerin ist. Dass ich von der "Akademikerklasse « spreche, ist zweifellos zugespitzt: Der Kern der Klasse hat einen akademischen Abschluss, aber nicht alle. Es gibt in der neuen Mittelklasse auch Personen ohne Hochschulabschluss (selbst wenn wir die Studierenden, also die aktuell an der Hochschule Ausgebildeten, außen vor lassen).

Man muss betonen: Das gilt zumal für Deutschland, wo aufgrund der Besonderheiten des Bildungssystems die Akademikerquote niedriger als in anderen westlichen Ländern ist. Kulturelles Kapital wird gerade in Deutschland auch jenseits der Hochschulen erworben. Darüber hinaus gibt es sicher biografische Sonderwege oder Gruppen mit Statusinkonsistenz. ${ }^{23}$ Ich vermute - aber auch hier müsste noch empirische Arbeit geleistet werden -, dass in Ländern wie den USA, Großbritannien und Frankreich die neue Mittelklasse noch eindeutiger eine Akademikerklasse ist, da dort die Bildungsexpansion und Akademisierung breiter sind. Man wird aber insgesamt gut daran tun, mit Schulzes Begriffen der "Kernbereiche", "Grenzzonen« und »Unschärfen« zu hantieren. Also: Der - auch institutionell einflussreichste - Kernbereich der neuen Mittelklasse wird heute in den westlichen Gesellschaften von Akademikerinnen und Akademikern gebildet, ${ }^{24}$ eine zweite Zone bilden Individuen mit hohem (nichtakademischen) formalen oder informel-

23 Dass nicht jedes Mitglied der neuen Mittelklasse akademisch gebildet ist, wird in der SINUS-Studie indirekt deutlich, da es in den vier genannten Milieus Personen gibt, die als mittlere Angestellte oder als Facharbeiterinnen und -arbeiter arbeiten. Ich denke allerdings, dass dieser Befund das Bild der neuen Mittelklasse zwar differenziert, aber auch nicht zu einem völlig anderen Bild führt. Überdurchschnittliches formales kulturelles Kapital muss (in Deutschland) nicht zwangsläufig Hochschulabschluss bedeuten. Heilpraktikerinnen oder Sozialarbeiter mit vielfältiger, aber in diesen Fällen nichtakademischer Ausbildung etwa werden sich bei SINUS bei den Sozial-Ökologischen wiederfinden, wenn sie die entsprechende kulturelle Orientierung haben. Und sie passen auch zu einem flexibilisierten Idealtypus neue Mittelklasse. Oder der Gründer eines Hamburger Szenerestaurants, der dafür eine Menge informelles kulturelles Kapital aufhäufen muss, wird sich bei SINUS unter den Expeditiven wiederfinden, wenn er die entsprechenden kulturellen Orientierungen hat - auch hier neue Mittelklasse, sofern die soziale Lage passt. Darüber hinaus gilt: Die soziale Realität ist vielgestaltig. Es mag auch Individuen geben, die tatsächlich kein überdurchschnittliches formelles oder beruflich verwertbares informelles kulturelles Kapital haben und die »gleichwohl « die entsprechenden Versionen des expressiven Individualismus leben.

24 Umgekehrt gilt: So wie nicht jedes Mitglied der neuen Mittelklasse einen akademischen Abschluss hat, so ist auch nicht jeder Akademiker und jede Akademikerin Teil des Werteclusters der neuen Mittelklasse, es gibt auch solche in der traditionellen Mittel- 
len kulturellen Kapital, und im weiteren Umfeld finden sich kapitalschwächere (auch kulturkapitalschwächere) Individuen. Sie alle eint aber eine ähnliche Weltsicht und Lebenspraxis des expressiven Individualismus.

\section{Die Geschichte der neuen Mittelklasse}

Ist die neue Mitteklasse nun wirklich so neu? Oder besser: Was an ihr ist neu, was nicht? Neben der Frage nach dem genauen Umfang dieser Klasse und ihrer internen Differenziertheit zweifeln Kumkar und Schimank vor allem meine These des historischen Wandels an. Ich habe in Das Ende der Illusionen diesen Wandel zugespitzt darstellt und bin an anderer Stelle - vor allem in meinem Buch Das bybride Subjekt ${ }^{25}$ - genauer auf die langfristigen Kontinuitäten und Diskontinuitäten der Lebensformen in der Geschichte der Moderne seit dem Ende des 18. Jahrhunderts eingegangen. Für mich ist es zentral, dass jede Transformation und Diskontinuität zugleich auf anderer Ebene mit Kontinuitäten oder besser: mit der Neuaneignung vergangener, historischer Elemente verbunden ist. Es gibt hier niemals komplette Brüche - das ist selbst ein Mythos des modernistischen Denkens -, sondern in den Brüchen immer zugleich Kontinuitäten. Dies gilt auch für den Modernisierungsprozess in der Mittelklasse.

Für ein Verständnis des Wandels der Mittelklasse scheint mir das historische Schema, das ich in Das bybride Subjekt entwickle und das die bürgerliche Moderne, die industriell-organisierte Moderne und die Spätmoderne als drei Phasen der Moderne unterscheidet, gut geeignet. Es wird dann deutlich, dass die kulturellen Muster, die sich in der neuen Mittelklasse finden, eine längere Vorgeschichte haben. Diese führt kulturelle Muster weiter, die sich im Doppel von Bürgerlichkeit (Selbstverantwortung, Bildungsinteresse, Ethik) und Romantik (Selbstverwirklichung, Ästhetik, Kreativität etc.) bereits in der bürgerlichen Moderne im 19. Jahrhundert fanden. ${ }^{26}$ Die neue Mittelklasse der Spätmoderne hat so neobürgerliche und sie hat neoromantische Züge. Insofern ergeben sich auch für die potenziell spannungsreiche Synthese aus Selbstverwirklichung und Statusinvestition in der neuen Mittelklasse durchaus Vorläufer im 19. Jahrhundert (»der Bürger, der Künstler sein will«).

Die Relation zwischen der neuen Mittelklasse der Spätmoderne und der breiten Mittelklasse der entfalteten industriellen Moderne der 1950er bis 1970er Jahre erweist sich ebenso als eine Kombination aus Kontinuitäten und Diskontinuitäten. Dafür muss man aber den Begriff der Mittelschicht oder Mittelklasse jenseits der Sozialstatistik als theoretisch gehaltvolles Konzept verstehen. Tatsächlich habe ich es als großen Fortschritt von Uwe Schimank, Steffen Mau und Olaf Groh-

klasse. Dass eine positive Korrelation zwischen dem expressiven Individualismus und der Hochschulbildung existiert, heißt nicht, dass beide Merkmale in jedem einzelnen Fall zusammen auftreten müssten.

25 Reckwitz 2020 [2006].

26 Vgl. auch Reckwitz 2017, S. 285-289. 
Samberg in ihrem Buch Statusarbeit unter Druck?27 gesehen, dass sie die »Mittelschicht " nicht bloß über eine sozialstatistisch bestimmbare soziale Lage definieren, sondern über eine bestimmte Logik der Lebensführung: In deren Zentrum steht investive Statusarbeit.

Das Muster der investiven Statusarbeit könnte man sicherlich wieder in das Bürgertum des 19. Jahrhunderts zurückverfolgen, aber in unserem Zusammenhang scheint es mir wichtig, dass diese Statusarbeit zum konstituierenden Merkmal der Mittelklasse nach 1945 wird, insbesondere in Westdeutschland und den USA (wo jeweils die Arbeiterschaft in der Mittelklasse aufgeht). Auch dies wäre sicherlich noch einmal einige genauere sozialhistorische Studien wert. Wenn dem so ist, dann greift aber die neue Mittelklasse, indem sie selbst fleißig investive Statusarbeit betreibt (davon gehe ich aus und das bestätigt auch SINUS im Wesentlichen), ${ }^{28}$ auf ein bereits existierendes Muster zurück, das nun modernisiert und perfektioniert wird (zum Beispiel dadurch, dass nun auch andere Kapitalsorten eine Rolle spielen, so das psychophysische Kapital). Das heißt aber: Der Bruch zwischen der neuen Mittelklasse und der umfassenden Mittelklasse der nivellierten Mittelstandsgesellschaft ist sicherlich nicht auf der Ebene der Statusinvestition zu suchen; er findet sich vielmehr auf der Ebene der Werte des expressiven Individualismus.

Tatsächlich nehme ich hier die Kulturrevolution um 1970 mit ihrer Counter Culture, die sich in einem massiven Kulturkonflikt mit der alten Mittelklasse manifestierte, als zentrale historische Gelenkstelle wahr, nach der sich etwas grundsätzlich verschiebt in der Kultur der Moderne. In einem historisch einmaligen Schub gewinnen nun die Selbstverwirklichungswerte - die sich historisch ihrerseits bis auf die Romantik zurückverfolgen lassen und die in der nivellierten Mittelstandsgesellschaft eher in gesellschaftlichen Nischen galten (Künstlermilieu, Jugendkulturen) - gesellschaftlich rasant an Bedeutung. Die Zahl derer, die sie vertreten, wächst stark - dies ist als Wertewandel seit Ronald Inglehart belegt. Diese Vertreterinnen und Vertreter des expressiven Individualismus mögen zu Anfang gegenkulturell orientiert sein, seit den 1980er Jahren handelt es sich jedoch um ein etabliertes, einflussreiches Segment, das sich danach auch ohne historischen Kontakt mit der Kulturrevolution der 1970er Jahre reproduziert und ausdehnt: eben um jene neue Mittelklasse, deren Entstehung durch die sozialen Rahmenbedingungen der Postindustrialisierung, des kognitiv-kulturellen Kapitalismus und der Bildungsexpansion begünstigt wird. Diese sozialen Rahmenbedingungen sind historisch in dieser Tragweite tatsächlich ebenfalls neu. Neu ist dann auch die mit der neuen Mittelklasse einhergehende beträchtliche Verbreitung einer Lebensform, in der Selbstentfaltung und Statusinvestition eine Synthese eingehen. Wie gut diese Synthese funktioniert, wie spannungsreich sie ist - das wäre in der Tat ein wichtiges, weiteres Thema für ein empirisches Forschungsprogramm »neue Mittelklasse«. Also: Es ist nicht alles neu in der neuen Mittelklasse, es fin-

27 Schimank et al. 2014.

28 Nur im Milieu der Sozial-Ökologischen scheint die Statusinvestition schwächer.

Leviathan, 49. Jg., 1/2021 
den sich Kontinuitäten kultureller Muster, aber die Konstellation nach 1970 ist sowohl kulturell als auch sozial in mehrfacher Hinsicht eine besondere.

\section{Die traditionelle/alte Mittelklasse}

Damit müssen wir nun aber doch noch einen Blick auf die alte oder traditionelle Mittelklasse werfen. Was macht diese aus? Wenn man nach einem Prototyp der Lebensführung der traditionellen Mittelklasse sucht, wird man bei den SINUSMilieus ebenfalls fündig. Man muss hier in jenes Milieu schauen, das sich tatsächlich genau in der Mitte der SINUS-Grafik befindet, das allerdings rein quantitativ nicht (mehr) besonders groß ist (in Deutschland 13 Prozent): das dort so genannte "Milieu der bürgerlichen Mitte". Es nimmt hinsichtlich seines sozialen Status (Einkommen, Beruf) eine mittlere Position ein. Entscheidend ist jedoch: Seine kulturellen Muster markieren eine deutliche Differenz zum expressiven Individualismus der vier Milieus der neuen Mittelklasse. SINUS (108) zählt die Merkmale auf: Das »Streben nach geordneten Verhältnissen, nach Harmonie [...] und Sicherheit - beruflich wie privat ", "[d]urch Zielstrebigkeit, Leistungs- und Anpassungsbereitschaft einen angemessenen Status in der Gesellschaft - in der wohlsituierten Mitte - erreichen ", »Treue und Stabilität " sind wichtige Werte, ebenso »Geborgenheit in einer intakten Familie "sowie der "Wunsch, auf den beruflichen und sozialen Status stolz sein zu können«. »Ordnung, Fleiß und Strebsamkeit « (111) sind zentral.

Die Distinktion gilt hier in zwei Richtungen: zu den Exzentrikerinnen nach oben, den Verlierern nach unten. SINUS macht zudem in diesem Milieu eine "zunehmende Verunsicherung und Furcht vor sozialem Abstieg ", "Angst, nicht mehr mitzukommen ", aus, die bis zu einer »Frustration über die herrschenden Verhältnisse (108) reichen kann. In dieser Milieucharakterisierung finden sich somit - abgesehen von den neuen, für die Spätmoderne typischen Verunsicherungen und Frustrationen - jene beiden Bündel von Lebensprinzipien, die man klassischerweise der Mittelschicht oder dem Mittelstand, jener großen Mitte, die einmal in den 1950er bis 1970er Jahren die "nivellierte Mittelstandsgesellschaft " ausmachte, zuschreiben kann: auf der einen Seite ein starker Wunsch nach Ordnung, Stabilität, Berechenbarkeit im eigenen Leben und der Gesellschaft insgesamt - der auch mit engen sozialen Bindungen im Nahbereich verknüpft sein kann -, auf der anderen Seite ein Statusinteresse und eine Orientierung an Lebenserfolg und kommodem Lebensstandard. Ordnung und Status - das ist die Doppelformel dessen, was die Mittelklasse traditionellerweise ausmacht. Selbstverwirklichung, Individualität, Authentizität, Experimentalismus, Flexibilität, Kosmopolitismus etc., die Werte des expressiven Individualismus, erscheinen hier denkbar fern.

Das größte Missverständnis, das Kumkars und Schimanks Aufsatz hinsichtlich meiner Arbeiten enthält, betrifft mein Verständnis der traditionellen Mittelklasse. Sie behaupten, dass in meinem Verständnis sich im Wandel von der industriellen Moderne zur Spätmoderne auch die Lebensprinzipien der alten Mittelklasse gewandelt hätten, ja wandeln müssten: »Logischerweise darf dann mindestens 
eine der heutigen beiden >Mittelklassen keine investive Statusarbeit mehr betreiben, sondern muss ihre Lebensführung ganz anders ausrichten - sonst gäbe es kulturell keinen Bruch. «29 Da ich der neuen Mittelklasse investive Statusarbeit zuschreibe und diese dort auch genauer unter die Lupe nehme, ${ }^{30}$ müsste ich der traditionellen Mittelklasse diese absprechen, so die Argumentation. Dies tue ich jedoch keineswegs und sehe auch nicht, warum ich dies "müsste «, im Gegenteil heißt es bei mir: "Statusinvestition, vor allem die Entwicklung ökonomischer Ressourcen, prägt diese Lebensform (der traditionellen Mittelklasse) «; ${ }^{31}$ hier ist » weiterhin das leitend, was den alten Mittelstand auszeichnete: die Doppelformel von Statusinvestition und Selbstdisziplin «. ${ }^{32}$

Mir scheint, dass Kumkar und Schimank hier eine der Pointen meiner Analyse verpasst haben. Entscheidend ist für meine Interpretation, dass sich die traditionelle Mittelklasse in ihrer Binnenstruktur gerade nicht wandelt, dass sich in ihr die Lebensprinzipien der 1950er bis 1970er Jahre im weiteren Verlauf vielmehr in wenig veränderter Weise reproduzieren, dass sich aber dadurch, dass die neue Mittelklasse (und im Übrigen auch die neue Unterklasse) entsteht, der Status der traditionellen Mittelklasse im gesamten sozialstrukturellen Gefüge wandelt. Aus der nahezu allumfassenden Mittelschicht der nivellierten Mittelstandsgesellschaft, die dort eine sozialkulturelle Hegemonie bezeichnete, ist jetzt eine "alte " Mittelklasse geworden, die sich in einer Sandwichposition zwischen neuer Mittelklasse und neuer Unterklasse wiederfindet. Gerade weil und indem sie relativ unverändert bleibt, wird sie gleichsam überholt von der partiell kapitalstärkeren und einflussreicheren »kulturellen Avantgarde« der neuen Mittelklasse. So kann sich durch diverse Effekte in der alten Mittelklasse die eigene Position - ob real oder "gefühlt « - im sozialstrukturellen Gefüge verschlechtern: Der Wertverlust mittleren Bildungskapitals angesichts der Akademisierung und der Statusverlust mancher ländlicher und kleinstädtischer Räume angesichts des ökonomischen Aufschwungs der Metropolregionen wären hier ebenso zu nennen wie die Liberalisierung im politischen Feld.

Ich habe mich selbst mit der Lebensform der traditionellen Mittelklasse bisher wenig systematisch befasst. Mein Interesse galt in Die Gesellschaft der Singularitäten dem sozialen Wandel und seiner Trägergruppe der neuen Mittelklasse. Man kann jedoch mit der SINUS-Studie die gleichfalls heterogene Binnenstruktur und zumindest den ungefähren Umfang der traditionellen Mittelklasse in der Gegenwartsgesellschaft abschätzen. Das Milieu der »bürgerlichen Mitte « markiert so etwas wie das soziale und kulturelle Zentrum der alten Mittelklasse, um das sich herum das "adaptiv-pragmatische Milieu " und das "konservativ-etablierte Milieu « sowie wohl zumindest teilweise das »traditionale Milieu « versammeln. Wie gesagt: Leider kann man mit SINUS nur bedingt auf Informationen zur sozia-

29 Kumkar, Schimank in diesem Heft, S. 20.

30 Vgl. Reckwitz 2017, S. 303-308.

31 Reckwitz 2019, S. 98.

32 Reckwitz 2017, S. 366; Hervorhebung im Original.

Leviathan, 49. Jg., 1/2021 
len Lage zurückgreifen. Die Studie erfasst vor allem nicht systematisch den Bildungshintergrund. Grafisch ordnet SINUS die bürgerliche Mitte teilweise der mittleren und teilweise der unteren Mittelschicht, das adaptiv-pragmatische Milieu der mittleren Mittelschicht zu, das traditionale Milieu hingegen reicht von der mittleren Mittelschicht bis in die Unterschicht hinein. Diese drei Milieus sind also im sozialen Status niedriger als die vier Milieus der neuen Mittelklasse, sie sind aber auf die gesamte Gesellschaft bezogen durchschnittlich. Anders das konservativ-etablierte Milieu: Dieses reicht von der mittleren bis in die obere Mittelschicht hinein und ist daher im sozialen Status - vermutlich auch im kulturellen Kapital - der neuen Mittelklasse vergleichbar. ${ }^{33}$

Die entscheidende Differenzlinie zwischen neuer und alter Mittelklasse ist jedoch eine kulturelle: Der Formel expressiver Individualismus und Statusinvestition auf der Seite der neuen Mittelklasse steht die Formel Ordnungsorientierung und Statusinvestition auf der Seite der alten Mittelklasse gegenüber. Neben der »bürgerlichen Mitte « lassen sich nun auch die kulturellen Prinzipien des »konservativ-etablierten Milieus « (10 Prozent der Bevölkerung) als eine geradezu lehrbuchartige Konkretion dieser Doppelformel lesen (»Erfolg durch Leistung, Pflichtbewusstsein, Zielstrebigkeit und Ernsthaftigkeit«, »Klassische Achievement-Orientierung auf einem Fundament konservativ-bürgerlicher Werte (23)) infolge des höheren sozialen Status hier aber mit starken Vorstellungen von »Hierarchie und Rang « verknüpft. So wie SINUS dieses Milieu charakterisiert, ist es anders als es Kumkar und Schimank andeuten - von Wertewandel in Richtung des expressiven Individualismus nicht betroffen, im Gegenteil: Die Conaisseurhaftigkeit der "Hyperkultur ", wie sie die neue Mittelklasse lebt, ${ }^{34}$ scheint sich in größtmöglicher Entfernung zum etabliert-konservativen Lebensstil dieses Milieus zu befinden. Kein Wunder, dass man sich gerade dort von der Hyperkultur häufig besonders herausgefordert sieht. Der Antagonismus »alte Kultur « gegen »neue Kultur« wird hier markant.

Das "adaptiv-pragmatische Milieu « (11 Prozent der Bevölkerung), das von SINUS als die jüngere Version der bürgerlichen Mitte präsentiert wird, setzt innerhalb der Doppelformel einen deutlichen Akzent auf die Statusinvestition (»ausgeprägte[r] Lebenspragmatismus", das "funktionale, utilitaristische Denken ", die »Identifikation mit der Leistungs- und Wettbewerbsgesellschaft « (91)), während die Ordnungsorientierung angesichts eines stärkeren Hedonismus hier blasser geworden ist. Spiegelbildlich dazu ist das »traditionale Milieu « (11 Prozent der Bevölkerung) ausgerichtet, ein Milieu vor allem von Personen im Ruhestand, die extrem auf Ordnung und Sicherheit setzen, ohne dass Statusinvestition noch eine größere Rolle spielen würde (»Festhalten an traditionellen Werten wie Pflichterfüllung, Anstand, Sparsamkeit, Ordnung und Disziplin « (142)) - die Grenzen zur Unterklasse scheinen hier fließend. Auch inmitten der alten Mittelklasse gibt es

33 Insofern gibt es ohne jeden Zweifel auch außerhalb der vier Milieus der neuen Mittelklasse Personen mit akademischem Abschluss, und zwar offenbar konzentriert im konservativ-etablierten Milieu.

34 Reckwitz 2017, S, 298-303. 
also zweifellos Heterogenität, die soziologisch interessant ist. Es handelt sich nicht um einen homogenen Block, und durchaus setzen hier an manchen Orten Modernisierungsprozesse ein, ${ }^{35}$ aber auch hier sollte man nicht vor lauter Bäumen den Wald übersehen. Alle diese Milieus exemplifizieren - so würde ich sie interpretieren - die traditionelle Mittelklasse-Formel von »Ordnung und Status ", sie alle stehen auf Distanz zum expressiven Individualismus der neuen Mittelklasse. Der entscheidende Lackmus-Test ist, dass sie alle entsprechende, mehr oder minder deutliche Distinktionen in dieser Richtung enthalten: die bürgerliche Mitte gegen »Exzentrik, Diversität « (111), die Konservativ-Etablierten gegen den »substanzlosen Zeitgeist « und den »fortschreitenden Werteverfall« (23), die Adaptiv-Pragmatischen gegen »Ideologen und Weltverbesserer, Unangepasstheit « (111), das traditionale Milieu gegen »Besserwisserei, Nachhaltigkeit, Intellektualität, Multikulti« (145).

Bei aller grundsätzlicher Differenz: Gibt es nun nicht trotzdem Gemeinsamkeiten zwischen den Milieus der neuen und der alten Mittelklasse? Dies ist tatsächlich eine wichtige Frage. Ganz grundsätzlich lassen sich solche Gemeinsamkeiten auch in meinem theoretischen Modell ausmachen - denn nicht ohne Grund ist ja in beiden Fällen weiterhin von der »Mittelklasse « die Rede. Das, was beide gemeinsam haben, darin folge ich exakt der Definition der Mittelschicht im Sinne von Schimank, Mau und Groh-Samberg: ${ }^{36}$ Mittelklasse ist da, wo investive Statusarbeit betrieben wird. Das differenziert die Mittelklasse von der Unter- wie von der Oberklasse. Die einzelnen Investitionsstrategien mögen sich unterscheiden, aber dass es im Leben zumindest auch immer darum geht, Status zu sichern und $\mathrm{zu}$ verbessern, das ist eine Gemeinsamkeit zwischen der neuen und der alten Mittelklasse. Wenn wir noch einmal auf das oben eingeführte Mehrebenenenmodell zu sprechen kommen, bewegen wir uns mit dem Feststellen dieser Homologie zwischen alter und neuer Mittelklasse auf der dritten, der übergreifenden Ebene, und es ist für bestimmte Fragestellungen in der Tat wichtig, diese Ebene in die Betrachtung miteinzubeziehen.

Ich denke, dass sich auf diese Weise auch der Befund der Fallstudie von Kumkar und Schimank hinsichtlich beruflicher Orientierungen in der Mittelklasse besser einordnen lässt. Ich kenne genaue Anlage und Reichweite dieser Fallstudie nicht.

35 Die Adaptiv-Pragmatischen scheinen in mancher Hinsicht selbst in Richtung »innenorientierter Modernisierung « zu streben. Es ist daher richtig, dass Kumkar und Schimank darauf hinweisen, dass es sich bei ihnen im Vergleich zur »bürgerlichen Mitte« um ein moderneres Milieu handelt. Aus gegenwärtiger Sicht scheint ihre Nähe zur traditionellen Mittelklasse aber doch deutlich größer als jene zur neuen. Man erkennt dies an ihrer spezifischen Distinktion in Richtung neuer Mittelklasse (siehe unten), zugleich ist ihr maßvoller »Hedonismus « (wie er sich ja auch in Teilen der Unterklasse findet und auf ästhetischen Schemata der Unterhaltung und Zerstreuung beruht) nicht das gleiche wie der »expressive Individualismus « (der mit einem starken Verständnis des Selbst arbeitet und so auch eine »Norm des Nonkonformismus " kultivieren kann). Dies ist ein kultursoziologisches Thema, das eine genauere Ausarbeitung verdienen würde.

36 Schimank et al. 2014.

Leviathan, 49. Jg., 1/2021 
Aber was die Typologie dreier beruflicher Orientierungen in der Mittelklasse angeht, die sich als Resultat der Untersuchung ergibt, würde ich Folgendes kommentieren: Richtig ist sicherlich, dass eine Gemeinschaftsorientierung des beruflichen Ethos eher für eine Zugehörigkeit zur traditionellen Mittelklasse und eine Berufsstolzorientierung eher für eine Zugehörigkeit zur neuen Mittelklasse spricht. Hinsichtlich jener Gruppe, für die Beruf mit investiver Statusarbeit verknüpft ist, lässt sich aber hinsichtlich ihrer Milieu- und Klassenzuordnung nicht wirklich eine Aussage machen. Denn Statusarbeit betreiben sie ja, in welcher Weise auch immer, alle: die Liberal-Intellektuellen und die bürgerliche Mitte, die Expeditiven und die Adaptiv-Pragmatischen, die neue und die alte Mittelklasse. Die Frage ist, welcher Stellenwert dieser Statusarbeit im Rahmen der kulturellen Logik der gesamten Lebensführung zukommt und mit welchen anderen Werten und Praktiken sie sich verbindet. Hier müsste man noch einmal genauer schauen. In jedem Fall ist aber trotz aller Differenzen die Statusarbeit eine Klammer, die alte und neue Mittelklasse verbindet, und dies deutlich gemacht zu haben ist möglicherweise ein Ergebnis der Fallstudie von Kumkar und Schimank.

\section{Kulturell-politische Konfliktlinien}

In der Fallstudie von Kumkar und Schimank scheint sich die kulturelle Differenzlinie zwischen neuer und alter Mittelklasse in Luft aufzulösen. Aus den besonderen Ergebnissen ihrer Studie wird man allerdings sicherlich nicht den allgemeinen Schluss ziehen können, es gebe schlichtweg den soziokulturellen und soziopolitischen Wandel nicht, der in jene Konfliktlinie mündet, dessen Auswirkungen wir von der Wahl Donald Trumps über das Brexit-Referendum bis zur Gelbwestenbewegung in Frankreich in den westlichen Gesellschaften der letzten Jahren beobachten konnten. Dass die Verunsicherung in der traditionellen Mittelklasse keine luftige Fiktion, sondern harte Realität ist, hat vielmehr eine Reihe von empirischen Untersuchungen, teils ihrerseits als Fallstudien, häufig aber auch in Form repräsentativer Untersuchungen, sehr deutlich gezeigt, auf internationaler Ebene wie auch auf Deutschland bezogen. ${ }^{37}$

37 Zweifellos: Auch hier muss man differenzieren, was die Ausbildung von Differenzen und Polarisierungen angeht. Auf einer ersten Stufe existieren "nur " soziokulturelle Differenzen "an sich « zwischen den Klassen: Man hat unterschiedliche Lebensprinzipien und lebt schlichtweg nebeneinanderher. Auf einer zweiten Stufe setzen sich die Differenzen "an sich " in Differenzen "an und für sich « um: Gruppen beginnen sich selbst im gesellschaftlichen Ganzen im Vergleich zu Anderen zu situieren, und namentlich jene, die sich benachteiligt sehen, beginnen Unzufriedenheit (Statusbedrohung, Bedrohung kultureller Dominanz) zu empfinden und Verantwortliche zu suchen. Es bildet sich so eine kulturelle Konfliktlinie aus. Auf einer dritten Stufe setzt sich diese ins politische Feld um: Sie wird dann als ein neuer Cleavage sichtbar, der sich etwa auf der Ebene von Parteipolitik und sozialen Bewegungen manifestiert. Erst auf dieser Stufe kann man von einer »Polarisierung " sprechen. 
Ich will nur exemplarisch ein paar davon nennen. Arlie Russel Hochschilds zu Recht hochgelobte Studie Strangers in Their Own Land, ${ }^{38}$ die auf qualitativen Interviews in Louisiana beruht, arbeitet sehr plastisch jene »deep story « des Systemmisstrauens heraus, wie sie sie bei ihren Interviewpartnerinnen und -partnern findet, die man der traditionellen (weißen) Mittelklasse in den kleinstädtischen Regionen der amerikanischen Südstaaten zuordnen kann. Es existiert hier eine grundsätzliche Unzufriedenheit, dass das soziale Aufstiegsversprechen für einen selbst nicht mehr gilt und man mit den eigenen kulturellen Mustern - die Hochschild als solche eines »rooted self" umschreibt - gesellschaftlich entwertet scheint. Zentral ist das Gefühl, in seiner Lebensleistung nicht anerkannt zu werden und von »den Anderen « übervorteilt zu werden, die scheinbar in der sozialen Hierarchie an ihnen vorbeiziehen - die Anderen, das sind die Menschen in den Metropolregionen, die Migrantinnen und Migranten, die erfolgreichen Frauen etc.

Nun mag man einwenden, dass es sich bei Hochschild »nur « um eine Fallstudie handelt und sie keine repräsentativen Aussagen für ein ganzes soziales Segment macht. ${ }^{39}$ Auch geht es hier um die USA, und man mag fragen, ob in Deutschland die Dinge nicht anders liegen. Die Milieustudie von SINUS enttäuscht jedoch auch diese Erwartung, und ich bin auf diesen Aspekt oben schon kurz eingegangen. Mehr oder minder deutliche Entwertungsgefühle und daraus folgende Ressentiments erweisen sich für weite Teile der traditionellen Mittelklasse (und - kaum verwunderlich - auch der Unterklasse, die hier nicht unser Thema ist) als charakteristisch. Für das Milieu der bürgerlichen Mitte ist so SINUS zufolge ein Selbstbild zentral, »Rückgrat der Gesellschaft, aber auch `Melkkuh der Nation« (112) zu sein, und hier gilt: »Krisengefühl [...] und Statusbedrohung [...] führen häufig zu einem Gefühl wachsender Überforderung und diffuser Frustration über die herrschenden Verhältnisse « (108). Das konservativ-etablierte Milieu braucht sich hinsichtlich seiner sozialen Lage sicherlich wenig Sorgen zu machen, aber hier sind für das Weltbild die »Bedenken hinsichtlich des fortschreitenden Werteverfalls « und die Sorge, dass »Meinungsführerschaft [...] aufgrund des raschen sozio-kulturellen Wandels verloren geht" (23), typisch. Für das traditionelle Milieu wird »Überforderung und Bedrohung durch den beschleunigten technologischen und sozialkulturellen Wandel« (142) als primäre Orientierung herausgearbeitet.

Man muss allerdings nicht beim SINUS-Institut bleiben, um zu solchen Ergebnissen zu kommen. Zieht man die ebenfalls repräsentative Untersuchung politischer Milieus und politischer Weltbilder zu Rate, die die Hans-Böckler-Stiftung unter der Überschrift Was verbindet, was trennt die Deutschen? Werte und Konfliktlinien in der deutschen Wählerschaft im Jahr 2017 veröffentlicht hat, kommt man - obwohl die Milieus dort etwas anders organisiert sind - zu vergleichbaren

38 Hochschild 2016.

39 Sehr aussagekräftig hinsichtlich der traditionellen Mittelklasse in den USA, insbesondere der weißen Arbeiterklasse, ist auch Joan Williams (2017).

Leviathan, 49. Jg., 1/2021 
Ergebnissen. ${ }^{40}$ Neun politische Milieus werden dort detailliert unterschieden, und auf abstrakterer Ebene kommen die Autoren zu dem Ergebnis, dass sich drei große Blöcke unterscheiden lassen: Auf der einen Seite steht der Block jener Milieus der gehobenen Mitte mit großem Systemvertrauen und Glauben in den Modernisierungsprozess. Das sind das »engagierte Bürgertum «, die »Kritische Bildungselite " und die mit der Marktwirtschaft Zufriedenen (43 Prozent). Dem stehen zwei Blöcke gegenüber, in denen Enttäuschung und Misstrauen groß sind: ein Block, der die »Verunsicherten " genannt wird und drei Milieus in der (mittleren bis gehobenen) Mittelklasse mit ca. 32 Prozent umfasst (verunsicherte Leistungsindividualisten, »Einzelkämpfer «, »Besitzstandswahrer«), und ein Block mit Milieus aus der unteren Mittelklasse und Unterklasse, der hier die »Enttäuschten « genannt wird (25 Prozent der Bevölkerung).

Natürlich: Man müsste im Detail schauen, wie genau sich die Milieudifferenzierung der Hans-Böckler-Stiftung wiederum in die Unterscheidung von neuer und alter Mittelklasse (sowie Unterklasse) übersetzt. Wichtig ist aber in jedem Fall, dass auch diese Untersuchung zeigt, dass die politische Konfliktlinie zwischen jenen, die sich - trotz aller Kritik - eins sehen mit dem gesellschaftlichen Fortschritt, und jenen, die meinen, in ihren Leistungen mangelhaft anerkannt zu werden und den Fortschritt an sich vorbeiziehen sehen, mitten durch jenes Segment der Gesellschaft geht, die man einmal »die Mittelklasse« genannt hat. Hier lassen sich keine feingliedrigen Interessenkonflikte zwischen vielen Milieus beobachten, sondern tatsächlich eine grundsätzliche Konfliktlinie.

Dies sind bemerkenswerte Ergebnisse, aber typisch deutsch sind sie nicht. Die kulturelle und zugleich politische Konfliktlinie, die mitten durch die einstmals große Mittelklasse hindurch geht, bestätigt sich auch in der umfangreichen ländervergleichenden, quantitativ-standardisierten Untersuchung von Pippa Norris und Ronald Inglehart. ${ }^{41}$ Auch sie machen einen Dualismus der Werte in der Bevölkerung aus und zeigen darüber hinaus, wie er sich ins politische Feld übersetzt. Als Reaktion auf die "silent revolution " von postmaterialistischen und sozialliberalen Werten hat sich Norris und Inglehart zufolge ein diametral entgegengesetzt ausgerichtetes Wertecluster konservativ-autoritärer, anti-liberaler Werte neuformiert (cultural backlash) und sich einen politischen Artikulationsraum insbesondere in Form des Rechtspopulismus geschaffen. Norris und Inglehart nennen verschiedene Faktoren, die beeinflussen, zu welchem Wertecluster man zählt: Der Faktor der Generation scheint dabei wichtig zu sein, aber auch die Bildung oder der Wohnort (Stadt, Land). ${ }^{42}$ In jedem Fall sind es Faktoren, die sich nicht auf rein materielle Unterschiede zurückführen lassen.

Die Analyse von Norris und Inglehart ist vielschichtig und lässt viele Fragen offen, zumal sie nur Korrelationen, aber keine Milieu- oder Klassendifferenzierung bieten. Aber in unserem Zusammenhang ist wichtig, dass sich in den europäischen und nordamerikanischen Gesellschaften die Entstehung und Wirkung einer

40 Siehe Müller-Hilmer, Gagné 2018.

41 Norris, Inglehart 2019.

42 Ebd., S. 113. 
neuen politisch-kulturellen Konfliktlinie zwischen sozialliberalen und konservativautoritären Werten empirisch gut belegen lässt, die mit der klassischen LinksRechts-Spaltung nicht identisch ist. Es handelt sich bei dieser Konfliktlinie nicht nur um ein Phänomen des politischen Feldes und der Ideologien von Parteien, vielmehr geht sie auch mitten durch die Wertestruktur der Wahlbevölkerung.

Die vier genannten Untersuchungen lassen sich zweifellos nicht ohne Weiteres in eins setzen. Auch sind sie nicht frei von sehr verschiedenen, einander widersprechenden normativen Tendenzen gegenüber den Werten und Erfahrungen der traditionellen Mittelklasse - zwischen Empathie und Pathologisierung -, an die ich nicht anschließen will. Aber die Indizien dafür, dass sich in der Spätmoderne eine neue kulturell-politische Konfliktlinie ausgebildet hat und dass diese sozialstrukturell eng mit der kulturellen Differenz zwischen neuer und alter Mittelklasse zusammenhängt, sind deutlich.

Damit sind nicht alle Fragen beantwortet, im Gegenteil. Die Gesellschaftstheorie formuliert nicht nur eine zugespitzte Synthese empirischer Beobachtungen, sie sollte auch in der Lage sein, Impulse für weitere empirische Untersuchungen zu liefern. Dafür nehme ich eine Reihe von Desiderata wahr, die sich in Auseinandersetzung mit Kumkar und Schimank herauskristallisiert haben: 1. Was lässt sich präziser über die Rolle des kulturellen Kapitals in den Milieus der neuen und jenen der alten Mittelklasse sagen, was über die Verteilung von Einkommen und Vermögen? 2. Lässt sich die These der kulturellen Hegemonie der Werte der neuen Mittelklasse - etwa über entsprechende Diskursanalysen für Felder wie Wirtschaft, Politik, Medien, Gesundheit etc. - empirisch plausibilisieren? 3. Welche nationalen Differenzen existieren in der Konstellation von neuer und alter Mittelklasse in verschiedenen westlichen Ländern, inwiefern unterscheidet sich hier Deutschland etwa von den USA, Großbritannien oder Frankreich? 4. In welchem Verhältnis stehen innerhalb der neuen Mittelklasse die Ziele des expressiven Individualismus und der Statusinvestition? 5. Kann man trotz aller Unterschiede die Gemeinsamkeiten zwischen neuer und alter Mittelklasse präziser bestimmen? Längerfristig wird man die weitere Dynamik der Klassenkonstellationen im Auge haben müssen. Denn weder die neue noch die alte Mittelklasse werden qualitativ oder quantitativ unverändert bleiben. Die zentrale Frage lautet dann: Gibt es Rekonfigurationen, neue Brüche oder neue Gemeinsamkeiten?

\section{Coda: Der Abwehrreflex der Soziologie gegen die neue Mittelklasse}

Abschließen möchte ich mit einer wissenssoziologischen Bemerkung zum Verhältnis der Soziologie zur neuen Mittelklasse. Diese Bemerkung kommt nicht ganz ohne leise Ironie aus. Wie gesagt: Die Entstehung, das Wachstum und der Bedeutungsgewinn einer häufig akademisch gebildeten sozialen Gruppe mit Werten des expressiven Individualismus ist bereits seit den 1970er Jahren ein Thema soziologischer Analyse, und zwar auf internationaler Ebene. Es ist vor diesem Hintergrund bemerkenswert, welchen Widerständen man namentlich in der deutschen Soziologie teilweise begegnet, wenn man sich für die Binnenstrukturen dieser 
Klasse und für ihre gesellschaftliche Relevanz interessiert (und damit ist nicht die differenzierte Position von Kumkar und Schimank gemeint).

Natürlich gilt dies nicht für alle: Gerade in Bereichen wie der Politischen Soziologie, der Kultursoziologie oder der Bildungssoziologie läuft man häufig offene Türen ein, wenn es um die Besonderheiten und Auswirkungen der neuen Mittelklasse geht. Aber bei nicht wenigen in unserer Zunft stößt man doch auf eine Skepsis, die mehr als nur sachliche Gründe zu haben scheint. Hin und wieder scheint man geradezu peinlich berührt oder legt eine Haltung allgemeinen Unverständnisses an den Tag. There is no such thing as a new middle class - dieses Credo ist so selten nicht. Manchmal wird man den Verdacht nicht los, dass versucht wird, die Relevanz der neuen Mittelklasse kleinzureden oder kleinzurechnen. Die einen mutmaßen, dass es sich hier lediglich um das grüne Milieu handele (nun, die Grünen haben bekanntlich auch schon Umfragewerte von 25 Prozent in Deutschland, aber sei's drum). Es müsse also um eine Art Prenzlauer-Berg-Soziologie gehen? Oder sind mit der neuen Mittelklasse urbane Fashion-Victims gemeint, die Hipster von Berlin-Neukölln? Man hat in der Zeitung schon davon gelesen, die soll es geben. Oder die Nachfahren der Yuppies der 1980er? Oder sind es die urbanen Kreativen? Auch für diese hat mancher eher ein müdes Lächeln parat, das kreative Prekariat, eh überschätzt! Oder soll es sich bei der neuen Mittelklasse um die mittlerweile notorischen kosmopolitischen Eliten handeln, von denen die Medien berichten, letztlich also lediglich eine zahlenmäßig winzige Elite, die gar nicht mehr zur Mittelklasse zählt? Ironisch zugespitzt, scheint für manche Soziologinnen und Soziologen zu gelten: Die neue Mittelklasse, das sind immer die anderen. Und wirklich wichtig ist sie nicht.

Man könnte diese Neigung zur vorschnellen Relativierung durchaus mit einer gewissen Leichtigkeit betrachten - neue Themen und Thesen stoßen eben immer wieder auf Widerstand, der nicht einfach in der Sache begründet ist, das ist im Routinebetrieb der Wissenschaft nicht unbedingt anders als in anderen Bereichen der Gesellschaft -, wenn es nicht eine durchaus ernste Angelegenheit wäre: Immerhin steht die Fähigkeit der Soziologie, die Gegenwartsgesellschaft zu begreifen, auf dem Spiel, und das ganz ohne Scheuklappen hinsichtlich dessen, wie man es gerne hätte. Es stellt sich die Frage: Was sind die Gründe und Ursachen für die soziologischen Abwehrreflexe gegen das Phänomen der neuen Mittelklasse? Ich vermute mehrere:

1. Wenn es um die Sozialstruktur geht, hat die zeitgenössische Soziologie vor dem Hintergrund ihres sozialkritischen Interesses eine Neigung, bevorzugt in die unteren Etagen der Gesellschaft zu blicken: in die prekäre Unterklasse, in die sozial absteigende Mittelklasse, in die Fraktionen der Modernisierungsverliererinnen und -verlierer. Sozialstrukturanalyse heißt im Kern Ungleichheitsanalyse, angeleitet von Maßstäben sozialer Gerechtigkeit. Wenn der Blick sich doch nach oben wendet, dann geht es eher um die Oberklasse der Superreichen, weil sich so eklatante Vermögensdifferenzen in der Gesellschaft demonstrieren lassen. Nun sind die Unter- und die Oberklasse zweifellos wichtige und untersuchungsbedürftige Phänomene, aber die Modernisierungsgewinnerinnen und -gewinner in der Mittelklasse geraten so systematisch aus dem Blick. Sich mit deren vermeintlichen 
Selbstverwirklichungsproblemen zu beschäftigen, scheint dann geradezu frivol. Warum sollte man sich mit den Gewinnern beschäftigen? Die Antwort ist allerdings einfach: um den sozialen Wandel und die neuen Konflikte zu verstehen.

2. Wenn man sich doch für die Mittelklasse interessiert, so ist dieses Interesse namentlich in Deutschland (ganz anders als in Großbritannien übrigens) bei manchen immer noch von der "Konsensfiktion Mittelklasse " der industriellen Moderne angeleitet. Es gibt hier das normative, bundesrepublikanische Ideal einer »breiten Mitte" vom Facharbeiter bis zum Ingenieur, das aber - so eine äußerst verbreitete Diagnose - in letzter Zeit von der » Krise der Mittelklasse « bedroht ist. Diese Krise der Mittelklasse interessiert die Soziologie also durchaus, aber wiederum vor allem unter dem negativen Aspekt der Statusbedrohung und Verlustangst in manchen Segmenten. Nun also von einer Dichotomie zwischen neuer und alter Mittelklasse auszugehen scheint alle Hoffnungen auf soziale Integration zunichte zu machen: Man würde quasi das amtlich machen, was man insgeheim verhindern will.

3. Die Differenz zwischen der neuen und der alten Mittelklasse ist in erster Linie keine materielle, sondern eine kulturelle Differenz (was sie nicht weniger machtvoll macht). Aber aller Rede vom cultural turn zum Trotz haben Teile der Soziologie häufig weiterhin ihre Probleme mit der Kultur als treibendem Faktor der Lebensführung, so scheint es. Müssen Klassen nicht eine materielle Grundlage haben? Sind Werte nicht verschleierte Interessen? Und wenn es doch um Kultur geht, dann eher mit Bourdieu im Gepäck: Kultur sei letztlich nicht mehr als ein Feld sozialer Distinktion, in dem die Herrschenden sich von den anderen symbolisch abgrenzen. Dass dies auch der Fall ist, ist sicher unbestritten. Aber dass Kultur mehr ist als Ideologie oder Distinktion, nämlich eine kulturelle Praxis mit ihren eigenen - teilweise von außen irrational erscheinenden - Wertigkeiten und als eine solche zu untersuchen ist, das scheint gar nicht so selbstverständlich zu sein. Wenn es um Sozialstruktur geht, ist immer noch relativ wenig Weber und viel Marx das gesunkene Kulturgut in großen Teilen der Soziologie. Phänomene der Ästhetik und der Ethik (das »schöne" und das "gute "Leben), die für die Lebensform der neuen Mittelklasse von großer Bedeutung sind, scheinen dann manchen als bloße Überbauphänomene höchstens sekundärer Relevanz, am Rande des soziologischen Interesses.

4. Die neue Mittelklasse ist politisch ein unsicherer Kantonist. Das macht sie schwierig, wenn man - wie weiterhin viele - von der Links-Rechts-Unterscheidung ausgeht. Ich würde vermuten, dass dieses unterschwellige politische Unbehagen eine nicht zu unterschätzende Rolle bei den analytischen Berührungsängsten gegenüber der neuen Mittelklasse spielt. Man muss nicht so weit gehen wie Nancy Fraser und das abschätzige Etikett des "progressiven Neoliberalismus" verwenden. ${ }^{43}$ Aber zweifellos und trotz aller Milieudifferenzen sprengt die neue Mittelklasse die Links-Rechts-Unterscheidung oder, anders formuliert, sie kombiniert Werte, die man eher Mitte-Links, und andere, die man Mitte-Rechts ansiedeln würde: Gleichberechtigung und Toleranz, Ökologie, Diversität einerseits,

43 Fraser 2017.

Leviathan, 49. Jg., 1/2021 
Selbstverantwortung, Flexibilität und Mobilität andererseits. Hinzu kommt: Die neue Mittelklasse ist gesellschaftlich einflussreich (oder gar hegemonial), und die Frage, ob dies nun eine gute oder eine schlechte Hegemonie ist, ist nicht einfach zu beantworten. Eingefahrene politische Reaktionen sind jedenfalls dadurch herausgefordert.

5. Das Phänomen der neuen Mittelklasse zwingt auch Soziologinnen und Soziologen zu einer Selbstbeobachtung, die durchaus unbehaglich sein kann. Die meisten von ihnen werden so wie die das Gros der Akademikerinnen und Akademiker zu den Milieus der neuen Mittelklasse zählen. Jene manchmal provozierende Selbstreflexion, welche die Soziologie ansonsten der Gesellschaft zumutet, müssen ihre Vertreterinnen und Vertreter nun auch auf die eigene, private Lebenswelt anwenden. Natürlich ist es bequemer, sich als "freischwebende Intellektuelle « (oder dessen kritische Verwandte, die solidarisch-parteiischen Intellektuellen) zu sehen. Und wenn die eigene Zuordnung in die Milieus und kulturellen Klassen schwerer fällt (man etwa Züge der »bürgerlichen Mitte « an sich entdeckt), macht dies die Situation nicht unbedingt komfortabler. Hinzu kommt: Der Frage nach der kulturellen Dominanz einer Klasse, der man selbst angehört, möchte man häufig lieber ausweichen. Und: Nimmt man nicht selbst in seinem privaten Leben unterschwellig an jenen sozialen Schließungstendenzen teil, etwa durch die Wahl von Schulen und Wohnvierteln, welche die neue Mittelklasse charakterisiert?

Natürlich: Alles ist noch komplizierter. Es gibt nicht nur einen soziologischen Abwehrreflex gegenüber der neuen Mittelklasse, der dazu führt, dass man dieses Phänomen unterschätzt, es gibt durchaus auch eine Dämonisierungstendenz und eine Glorifizierungstendenz, die unter Umständen dazu führen können, dass man die neue Mittelklasse übermäßig fokussiert. Auch diese muss man benennen. Die Dämonisierungstendenz ist im politischen und medialen Raum stärker als im wissenschaftlichen: Für manche scheint die neue Mittelklasse mittlerweile zu einer Art Sündenbock dafür geworden zu sein, was in der spätmodernen Gesellschaft alles falsch läuft. Die neue Mittelklasse als eine Gruppe von Egozentrikern und Egoistinnen, wahlweise eine »loony left « oder aber der Hort des Neoliberalismus oder beides. Die »Kosmopoliten « sind mittlerweile zu einem Feindbild nicht nur auf der Neuen Rechten, sondern auch für manche in der selbstbewusst populistischen Linken geworden. Publizistisch ist David Goodharts ${ }^{44}$ Gegenüberstellung der vaterlandslosen anywheres gegenüber den solide-berechenbaren somewheres, in der die Wertigkeiten eindeutig zuungunsten der neuen Mittelklasse verteilt sind, besonders wirkungsmächtig. Aus dieser Sicht gilt: Man braucht die neue Mittelklasse als Zielscheibe, ja als Gegner des populistischen Antagonismus zwischen Volk und Eliten.

Ein spezieller Fall ist hier die Fraktion jener, die sich die Selbstkritik und das schlechte Gewissen zur Routine gemacht haben: »Ja, wir sind neue Mittelklasse, und haben wir es nicht wirklich übertrieben? " Auch eine solche Selbstbeschämung kann identitätsstiftend wirken - denn aus der selbstkritischen Haltung vermag man unter Umständen doch wieder erhebliches Selbstbewusstsein zu ziehen - 
und insofern das Interesse an der neuen Mittelklasse anheizen. Erst recht gilt letzteres für jene, für die die neue Mittelklasse wichtig ist, da sie ihnen im positiven Sinne als Avantgarde des sozialen Wandels erscheint. Aus dieser Sicht hofft man auf ihre Stärke: Richard Florida setzte in The Rise oft the Creative Class zweifellos große Hoffnungen auf die Liberalität und Offenheit der "kreativen Klasse", und für Ronald Inglehart ist schon seit den 1970er Jahren der Wertewandel ein Pendant zum »Marsch durch die Institutionen " - ein Zeichen dafür, dass die Kulturrevolutionäre von gestern glücklicherweise die Dominanz errungen haben. Diese Glorifizierungstendenzen waren allerdings in der Soziologie bisher eher schwach, aber es gibt sie, und auch hier muss man Vorsicht walten lassen. Insgesamt gilt: Das Phänomen der neuen Mittelklasse ist ein umstrittenes Feld, ein vermintes, ein politisiertes Gelände, in dem deutlich mehr als nur wissenschaftliche Neugier und Wahrheitssuche verhandelt wird. Und in der deutschen Soziologie führt diese Gemengelage tendenziell dazu, die Bedeutung der neuen Mittelklasse $\mathrm{zu}$ unterschätzen und das Interesse für Analysen ihrer speziellen Lebensform zu minimieren.

Es ist also durchaus kompliziert. Weder kann es darum gehen, die neue Mittelklasse zu verdammen, noch darum, sie zu glorifizieren. Es geht vielmehr darum, sie in ihrer gesellschaftlichen Relevanz für den sozialen Wandel und die Konfliktlagen der Spätmoderne ernst zu nehmen. Ich hoffe, es gelingt uns in der Soziologie, die neue Mittelklasse zu dem zu machen, was sie sein sollte: der Gegenstand eines theoretisch-empirischen Forschungsprogramms.

\section{Literatur}

Arsel, Zeynep; Bean, Jonathan 2013. »Taste Regimes and Market-Mediated Practice«, in Journal of Consumer Research 39/5, S. 899-917.

Bell, Daniel 1973. The Coming of Post-Industrial Society: A Venture in Social Forecasting. New York: Basic Books.

Bourdieu, Pierre 1989. Die feinen Unterschiede. Kritik der gesellschaftlichen Urteilskraft. Frankfurt a. M.: Suhrkamp.

Currid-Halkett, Elizabeth 2017. The Sum of Small Things. A Theory of the Aspirational Class. Princeton: Princeton University Press.

Featherstone, Mike 1991. Consumer Culture and Postmodernism. London et al.: Sage.

Florida, Richard 2002. The Rise of the Creative Class. And How It's Transforming Work, Leisure, Community and Everyday Life. New York: Basic Books.

Fraser, Nancy 2017. "Vom Regen des progressiven Neoliberalismus in die Traufe des reaktionären Populismus ", in Die große Regression. Eine internationale Debatte über die geistige Situation der Zeit, hrsg. v. Geiselberger, Heinrich, S. 77-92. Berlin: Suhrkamp.

Goodhart, David 2017. The Road to Somewhere. The Populist Revolt and the Future of Politics. London: Hurst \& Co.

Harrison, Rob et al. Hrsg. 2005. The Ethical Consumer. London: Sage.

Hochschild, Arlie Russell 2016. Strangers in Their Own Land. Anger and Mourning on the American Right. New York: The New Press.

Inglehart, Ronald 1977. The Silent Revolution. Changing Values and Political Styles Among Western Publics. Princeton: Princeton University Press.

Lareau, Anette 2003. Unequal Childhoods. Class, Race, and Family Life. Berkeley: University of California Press.

Leinberger, Paul; Tucker, Bruce 1991. The New Individualists. The Generation after the Organization Man. New York: Harper Collins.

Leviathan, 49. Jg., 1/2021 
Müller-Hilmar, Rita; Gagné, Jérémie 2018. Was verbindet, was trennt die Deutschen? Werte und Konfliktlinien in der deutschen Wählerschaft im Jahr 2017. Düsseldorf: Hans-Böckler-Stiftung. https://policy-matters.de/site/assets/files/1287/p_fofoe_report_002_2018.pdf (Zugriff vom 03.01.2021).

Norris, Pippa; Inglehart, Ronald 2019. Cultural Backlash. Trump, Brexit and Authoritarian Populism. Cambridge: Cambridge University Press.

Putnam, Robert 2015. Our Kids. The American Dream in Crisis. New York: Simon \& Schuster.

Reckwitz, Andreas 2017. Die Gesellschaft der Singularitäten. Zum Strukturwandel der Moderne. Berlin: Suhrkamp.

Reckwitz, Andreas 2019. Das Ende der Illusionen. Politik, Ökonomie und Kultur in der Spätmoderne. Berlin: Suhrkamp.

Reckwitz, Andreas 2020 [2006]. Das bybride Subjekt. Eine Theorie der Subjektkulturen von der bürgerlichen Moderne zur Postmoderne. Berlin: Suhrkamp.

Reckwitz, Andreas; Rosa, Hartmut 2021. Spätmoderne in der Krise. Was leistet die Gesellschaftstheorie? Berlin: Suhrkamp (im Erscheinen).

Schimank, Uwe; Mau, Steffen; Groh-Samberg, Olaf 2014. Statusarbeit unter Druck? Zur Lebensführung der Mittelschichten. Weinheim: Beltz Juventa.

Schulze, Gerhard 1992. Die Erlebnisgesellschaft. Kultursoziologie der Gegenwart. Frankfurt a. M.: Campus.

Strenger, Carlo 2019. Diese verdammten liberalen Eliten. Wer sie sind und warum wir sie brauchen. Berlin: Suhrkamp.

Wheaton, Belinda 2013. The Cultural Politics of Lifestyle Sports, London: Routledge.

Williams, Joan 2017. White Working Class. Overcoming Class Cluelessness in America. Boston: Harvard Business Review Press. 
Zusammenfassung: Der Aufsatz antwortet auf die Auseinandersetzung von Nils Kumkar und Uwe Schimank mit Andreas Reckwitz' Theorie der neuen und der alten Mittelklasse. Im Zuge dessen werden das Abstraktionsniveau, die historische Entwicklung und der Umfang der beiden Klassen geklärt. Detailliert werden die Milieustudien des Heidelberger SINUS-Instituts für die Analyse der neuen und alten Mittelklasse ausgewertet. Der Aufsatz endet mit einer Diskussion der Ursachen für die »Abwehrreflexe« der Soziologie gegenüber der neuen Mittelklasse.

Stichworte: Sozialstruktur, Milieus, Mittelschicht, Lebensstil, Kultur

\section{Where and Who Is the »New Middle Class «? A Reply to Nils Kumkar and Uwe Schimank}

Summary: The article responds to Nils Kumkar's and Uwe Schimank's critical debate of Andreas Reckwitz's distinction between the new and the old middle class. The level of abstraction, the historical development and the scope of the two classes are clarified. The model of social milieu of the Heidelberg SINUS Institute is evaluated in detail for an analysis of the new and the old middle class. The article ends with a discussion of possible causes for the "defensive reflexes " of German sociology to the new middle class.

Keywords: social structure, classes, middle class, lifestyle, culture

Autor

Andreas Reckwitz

Humboldt-Universität zu Berlin

Institut für Sozialwissenschaften

Unter den Linden 6

10099 Berlin

Deutschland

andreas.reckwitz@hu-berlin.de

Leviathan, 49. Jg., 1/2021 